\title{
The MGuard coronary stent: safety, efficacy, and clinical utility
}

\author{
This article was published in the following Dove Press journal: \\ Vascular Health and Risk Management \\ 18 September 2015 \\ Number of times this article has been viewed
}

\section{Montserrat Gracida \\ Rafael Romaguera \\ Francisco Jacobi \\ Joan A Gómez-Hospital \\ Angel Cequier}

Heart Diseases Institute, Hospital Universitari de Bellvitge - IDIBELL, University of Barcelona, Barcelona, Spain
Correspondence: Rafael Romaguera Heart Diseases Institute, Hospital Universitari de Bellvitge - IDIBELL, University of Barcelona, Feixa llarga s/n, L'Hospitalet de Llobregat, 08907 Barcelona, Spain

Tel +34932607539

Fax +3493260754

Email rafaromaguera@gmail.com
Abstract: Atheromatous and thrombotic embolization during percutaneous coronary revascularization is a feared complication that may cause impaired myocardial reperfusion even with a patent epicardial vessel. The MGuard stent is a cobalt chromium bare metal stent with a porous net attached to its outer surface that has been designed to prevent thrombus fragmentation and distal embolization during stent implantation. This review summarizes the available evidence supporting the use of the MGuard stent in different scenarios such as lesions with high thrombus burden, saphenous vein graft interventions, coronary perforations, or carotid lesions.

Keywords: Coronary artery disease, myocardial infarction, coronary stent, complication, thrombus, no-reflow phenomenon

\section{Introduction}

Atheromatous and thrombotic embolization during percutaneous coronary intervention (PCI) is a feared complication that may cause impaired myocardial reperfusion even with a patent epicardial vessel. ${ }^{1}$ Patients with acute coronary syndromes and those undergoing PCI of saphenous vein graphs (SVGs) are at highest risk of distal embolization, which frequently occurs after stent implantation due to thrombus fragmentation and protrusion by the stent struts. Inadequate myocardial reperfusion may occur in up to $50 \%$ of cases despite the use of optimal evidence-based coronary revascularization and is associated with a worse in-hospital and long-term prognosis. ${ }^{2}$

Prevention of embolization rather than treatment should be the way forward as treatment is unlikely to succeed if no reflow is established. Thus, several pharmacological and technical strategies have been tested to minimize the distal embolization risk. ${ }^{3}$ Distal protection devices failed to show reductions in infarct size or mortality in patients undergoing primary PCI, ${ }^{4}$ although they seem to improve angiographic and clinical outcomes in PCI of degenerated SVG. ${ }^{5}$ Similarly, catheter-based thrombus aspiration has been associated with controversial results; although early single-center studies suggested a potential benefit, recent trials have shown no reductions in infarct size or mortality. ${ }^{6,7}$

The concept of a stent covered with a membrane attached to its outer surface (to create a mechanical barrier to prevent thrombus fragmentation and filtration through the struts) has also been tested previously. ${ }^{8}$ However, covered stents, compared with bare metal stents, were associated with an increased risk of myocardial infarction, thus prohibiting their use in this scenario. Importantly, the barrier created by the membrane was related to delayed endothelization of the stent and also to side branch occlusion, which could explain the increased rate of myocardial infarction. ${ }^{9}$ 
The MGuard stent is a novel bare metal stent with a porous net attached to its outer surface that has been designed to trap thrombus debris reducing distal embolization, while pores allow normal endothelization of the stent through the membrane. The purpose of this review is to summarize all the relevant literature available about the use of this device.

\section{The MGuard stent}

The MGuard ${ }^{\mathrm{TM}}$ stent consists of a balloon-expandable closecell design bare metal stent with a polyethylene terephthalate microfiber sleeve attached to its outer surface (Figure 1). The first-generation stent used a 316L stainless steel frame with strut thickness of $100 \mu \mathrm{m}$, while the new MGuard ${ }^{\mathrm{TM}}$ Prime platform is manufactured of L605 cobalt chromium alloy (strut thickness $80 \mu \mathrm{m}$ ). The Micronet ${ }^{\mathrm{TM}}$ is identical on both stents, with a fiber width of $20 \mu \mathrm{m}$ and an expanded aperture size of $150 \times 180 \mu \mathrm{m} .{ }^{10}$ Thus, the net creates custom-designed pores that are 5- to 40-fold smaller than the stent cells and appears to effectively act as a mechanical barrier decreasing or even preventing the thrombus protrusion and embolization to the distal vessel. ${ }^{11}$ MGuard $^{\mathrm{TM}}$ and MGuard ${ }^{\mathrm{TM}}$ Prime diameters range from $2.5 \mathrm{~mm}$ to $4.0 \mathrm{~mm}$, while lengths range from 11 to 39 and 13 to $38 \mathrm{~mm}$, respectively.

\section{Technical considerations Guiding catheter, deliverability, and stent dislodgement}

The MGuard stent is compatible with 0.014 in guide wires and $6 \mathrm{Fg}$ guiding catheters. Crossing profile is slightly higher than newer-generation bare metal stents $(1.1-1.3 \mathrm{~mm}$ for the stainless steel platform and 1.0-1.2 mm for the cobalt chromium platform), which together with the close-cell design may impair the deliverability. In a recent trial, the MGuard stent was unable to reach or cross the culprit lesion in nine of 217 cases $(4.1 \%) .^{12}$ Of note, all nine cases occurred with the stainless steel platform. Similarly, a recent single-center experience reported $1.8 \%$ of cases in which the stent could not reach the lesion. ${ }^{13}$

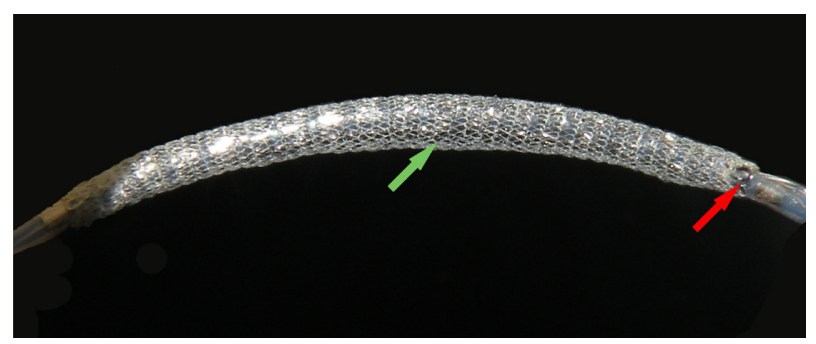

Figure I MGuard stent crimped onto a balloon.

Notes: Green arrow indicates the mesh sleeve. Red arrow indicates the metallic platform.
From January 2011 to April 2014, the manufacturer received 54 complaints of stent dislodgement from the delivery system, most occurring with the MGuard Prime stent. By contrast, no cases of net detachment from the stent were seen. Stent dislodgement was reported in two of 217 cases $(0.9 \%)$ of the prospective, randomized, multicenter evaluation of a polyethylene terephthalate micronet mesh-covered stent (MGuard) in ST-segment elevation myocardial infarction (MASTER) trial. ${ }^{12}$ Similarly, stent dislodgement occurred in six of 155 cases with the MGuard Prime stent (3.87\%) at the IDE-MGuard for acute ST-elevation reperfusion II (MASTER II) trial, ${ }^{14}$ which led the manufacturer in 2014 to a voluntary field safety corrective action. This included the temporary suspension of the MASTER II trial and the addition of a new step during the manufacturing process socalled thermoforming in which the balloon-shaping process forms "shoulders," which are intended to provide additional securement of the stent to the delivery system.

\section{Side branch occlusion}

Due to its double-layer design (metallic platform + mesh sleeve fibers), the MGuard stent may increase the risk of side branch (SB) occlusion. SB compromise may be related to two mechanisms: first, the summation of both layers (the metallic struts and the sleeve fibers) may act as a mechanical barrier blocking the coronary flow to the SB; second, the thrombus nearly located to the bifurcation may shift from the main vessel to the SB. It is possible that SBs compromised by the second mechanism could slightly improve with pharmacological treatment. Nevertheless, if the occluded SB is clinically relevant, a bifurcation technique may be considered (Figure 2). In our experience, wiring the SB should not be extremely complicated. By contrast, to advance a balloon across the net may require additional support measures such as the use of extension catheters.

\section{MGuard stent in patients with ST-segment elevation myocardial infarction}

After promising preliminary results in two small studies, efficacy and safety of the MGuard stent in patients undergoing primary PCI were first assessed by Piscione et al ${ }^{15}$ (Table 1). This was a multicenter Italian prospective registry that included 100 patients with ST-segment elevation myocardial infarction (STEMI). Thrombectomy was performed in 10\% of the patients and predilation in $42 \%$ of cases. Complete myocardial reperfusion including myocardial blush grade $=3$ and ST-segment resolution at 60 minutes was achieved in 90\% 


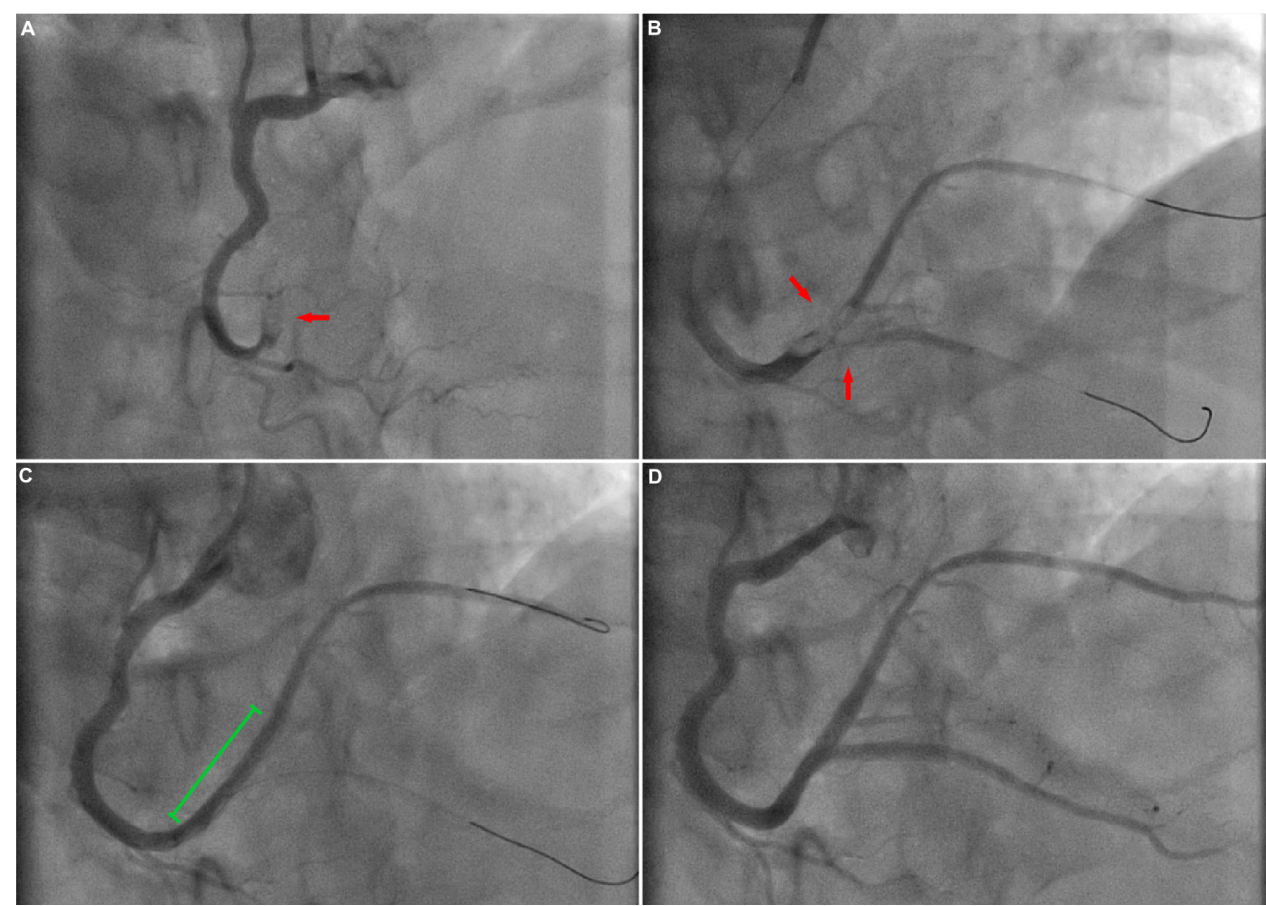

Figure 2 Coronary angiogram ( $30^{\circ}$ cranial oblique projection) showing a totally occluded right coronary artery before intervention (A). Despite exhaustive thrombus aspiration. Red arrow points to the thrombotic total occlusion. (B), a high thrombus burden (TIMI thrombus score $=4$ ) persisted at the bifurcation (red arrows). After MGuard deployment (green line), TIMI 3 flow was restored to the posterolateral branch, although the posterior descending artery persisted totally occluded (C). Following "reverse T stenting," final TIMI flow was 3 in both the posterior descending artery and the posterolateral branch branches (D, final result).

Abbreviation: TIMI, thrombolysis in myocardial infarction.

of patients. In hospital mortality occurred in seven patients (five presenting with cardiogenic shock preprocedure). Two subacute stent thromboses were also reported. Major adverse cardiovascular events (MACEs) up to 2 years were observed in $7.9 \%$ of the patients. Similarly, the MAGICAL study was a prospective multicenter international trial that enrolled 60 consecutive patients with STEMI. ${ }^{16}$ In this study, a high rate of complete epicardial and myocardial perfusion was also achieved (thrombolysis in myocardial infarction [TIMI] 3 flow in $93 \%$ of patients, myocardial blush grade 3 in $73 \%$, ST-segment resolution in 68\%). Cardiac mortality up to 3 years was very low $(7 \%) .{ }^{17}$

As opposed to previous studies in whom manual aspiration was performed in $<20 \%$ of cases, our group first reported results of the MGuard stent in selected cases of failed manual aspiration. ${ }^{13}$ The study included 56 consecutive patients in whom a high thrombus burden persisted after aggressive manual aspiration. After MGuard stent deployment, $>89 \%$ of cases had thrombus score $=0$, and $>70 \%$ ST resolution was observed in $58.7 \%$ of patients. Only one stent thrombosis (acute) was reported, and 9-month MACE rate was very low $(3.6 \%)$.

Importantly, a recent retrospective study compared results of consecutive procedures performed with either bare metal stents or MGuard stents. ${ }^{18}$ Due to differences in baseline characteristics between groups, a propensity matching was performed. After adjustment, patients treated with the MGuard stent presented with restenosis more frequently than those treated with bare metal stents. These results, however, have not been confirmed in other recent studies. ${ }^{19}$

Two large-scale randomized trials have compared the MGuard stent to conventional PCI. The MASTER I trial was a prospective, multicentre, international, randomized study in which 433 subjects presenting with STEMI were randomized in a 1:1 fashion to the MGuard stent versus bare metal or drug-eluting stents. ${ }^{12}$ Device success was greater in the control arm (95.9\% for MGuard vs $99.1 \%$ for control; $P=0.003$ ), but better reperfusion indices were obtained in the study group, as shown by the primary endpoint of the study, ST segment resolution ( $57.8 \%$ vs $44.7 \% ; P=0.008)$, and incidence of TIMI 3 flow ( $91.7 \%$ vs $82.9 \%, P=0.006)$. Consequently, mortality at 30 days ( $0 \%$ vs $1.9 \%, P=0.06)$ and 1 year ( $1 \%$ vs $3.3 \%, P=0.09)$ tended to be lower in the MGuard group. Subgroup analyses showed the highest differences favoring the MGuard stent in cases with large thrombus burden ${ }^{20}$ or long delay time. ${ }^{21}$ However, incidence of repeat revascularization was higher than the control group $(8.6 \%$ vs $0.9 \%, P=0.0003) .{ }^{22}$ The MASTER II trial was an international, multicenter, randomized trial designed to enrol 1,114 patients to show superiority of the MGuard stent vs 


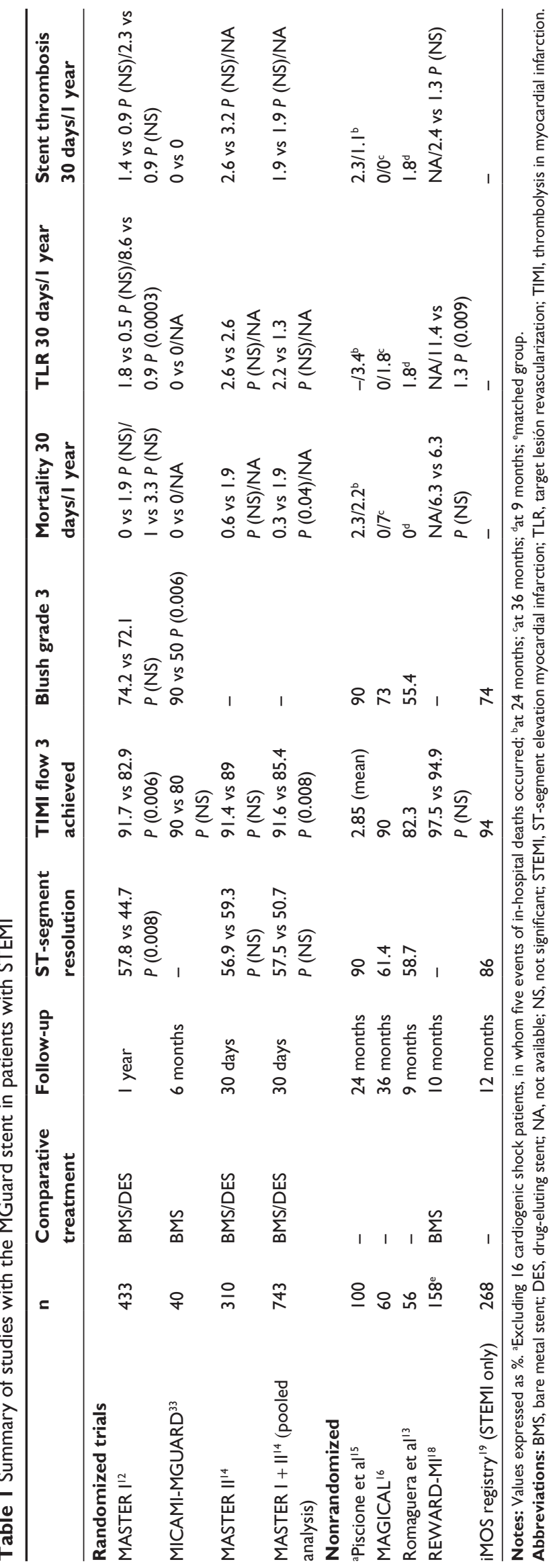

conventional PCI in clinical events such as death or myocardial infarction. However, due to a higher than expected frequency of stent dislodgement, the study was voluntarily suspended in April 2014 after enrolment of 310 patients (155 patients per group). Analysis of the enrolled cohort showed no benefit of the MGuard stent in any of the analysed endpoints (ST-segment resolution was $56.9 \%$ vs $59.3 \%$ in the control group, $P=0.68$; mortality was $0.6 \%$ vs $1.9 \%$ in the control group, $P=0.62) .{ }^{14}$ Similarly, a pooled analysis of the MASTER I and II trials ( $\mathrm{n}=743$ ) failed to show better myocardial reperfusion (ST-segment resolution 57.5\% for the MGuard vs $50.7 \%$ for the control group, $P=0.07$ ), although mortality at 30 days was lower $(0.3 \%$ vs $1.9 \%, P=0.03) .{ }^{14}$

Thus, data from randomized trials suggest that the use of MGuard stent may reduce distal embolization and may improve survival at 30 days compared with other commercially available coronary stents, although further randomized studies are warranted to confirm this hypothesis. Also, further research is needed to understand if the restenosis rate is only related to the lack of drug elution or, by contrast, if there is also an antigenic stimulus caused by the polyethylene terephthalate mesh. ${ }^{23}$

\section{MGuard stent in saphenous vein grafts}

Compared with atherosclerotic plaques in native coronary arteries, SVG plaques are softer, longer, more friable, and contain necrotic debris and cholesterol crystals. Thus, percutaneous revascularization of SVG is associated with high rates of distal embolization, no-reflow phenomena, and periprocedural myocardial infarction. To avoid this complication, several drugs and devices have been tested. Graft stents have shown discouraging results and distal protection devices, although effective, are associated with an increase of procedural length and costs. ${ }^{24,25}$

The performance of the MGuard stent has been tested in two small multicenter prospective registries. The first-inman study included 29 patients (17 with SVG intervention, mean SVG age $=12.6$ years $).{ }^{26}$ Device and procedural success was $100 \%$. Final TIMI 3 flow was observed in all cases, and periprocedural myocardial infarction was observed in a single case. However, an extension of the study with 23 SVG interventions showed a high rate of MACE (23\%), although no stent thrombosis or cardiac deaths were observed up to 20 months of follow-up. ${ }^{27}$ Similarly, the INSPIRE trial ${ }^{28}$ reported a high rate of procedural success of the MGuard stent in 16 SVGs, achieving final TIMI 3 flow in all cases. Nevertheless, in agreement with the previous study, a high 
frequency of repeated revascularization of the target vessel was observed at 12 months of follow-up (19\%).

\section{MGuard stent in other scenarios Coronary perforations}

Graft stents seem to be an effective tool to seal severe perforations that have persisted despite prolonged balloon inflation. However, the high profile and low flexibility of previous covered stents compromise the deliverability of these devices, particularly in emergency situations. ${ }^{9}$ Moreover, the mechanical barrier created by the membrane may preclude the endothelization of the stent, which eventually may be associated with stent thrombosis or with restenosis at the stent edges. Our experience, although limited, suggests that the MGuard stents can also be successfully used to seal coronary perforations. ${ }^{29}$ The net, although pored, appears to effectively act as a mechanical barrier, decreasing or even preventing the leakage to the pericardial cavity. Nevertheless, additional mechanism might be involved, such as the stretching of the vessel or the compression of the disrupted tissue. Moreover, the cobalt chromium platform would be more deliverable than the graft stent allowing also successful deployment in tortuous vessels, whereas the porous net would allow endothelization of the stent. Importantly, when MGuard stents are used in this bailout situation, high rates of restenosis may be expected. The mechanism, although unknown, might also be related to the inflammatory process involving the perforation.

\section{Coronary aneurysms}

Coronary artery aneurysms are a rare condition with a reported incidence of $0.14 \%-4.9 \%$ of patients undergoing coronary angiography and $0.3 \%-5.3 \%$ in patients after PCI. The majority of coronary aneurysms are atherosclerotic in origin and, in most cases, they are asymptomatic. However, potential complications associated with these abnormalities include thrombus formation, distal embolization, and, rarely, rupture.

Current available evidence for MGuard stent is limited to two case reports, ${ }^{30,31}$ in which the MGuard stent was successfully implanted, achieving complete exclusion of the aneurysm. However, the percutaneous exclusion of coronary aneurysm is not generally recommended, and therefore the use of the MGuard stent in this scenario should not be advised. Moreover, in our experience, although limited to two cases, MGuard stent implantation did not achieve total exclusion of the aneurysm in the early phase and was related to severe restenosis at late follow-up.

\section{Carotid arteries}

To avoid microembolization after stent implantation in carotid artery stenosis, a mesh-covered stent may be implanted. Notably, for carotid arteries, the manufacturer has mounted the same porous net over a nitinol self-expandable platform (so-called CGuard stent). Although the clinical experience is very limited, recent results of the CARENET trial have shown a very low rate of new ischemic ipsilateral lesions (as assessed by diffusion-weighted magnetic resonance imaging) as well as no major adverse cardiac and cerebrovascular events at 30 days. ${ }^{32}$ However, although these results are promising, more data are needed before making recommendations about the safety/efficacy of this device.

\section{Perspectives}

Considering the clinical benefits associated with drug-eluting stents in patients with STEMI and also due to the relatively high rate of restenosis of the MGuard stent, the manufacturer decided in 2014 to definitely cancel the MASTER II trial and to start development of the drug-eluting version of the MGuard stent. This new device will elute sirolimus from the stent platform (rather than the net) and will be available in late 2015.

\section{Conclusion}

The MGuard stent is a useful tool to avoid distal embolization during PCI of thrombotic lesions. The micronet attached to its outer surface prevents thrombus fragmentation and filtration during stent deployment. The enhanced myocardial reperfusion achieved with the use of the MGuard stent may be associated with a reduced mortality compared with other commercially available bare metal or drug-eluting stents. However, its use is associated with some limitations that must be highlighted: 1) the stent deliverability (even with the cobalt chromium version) may be slightly worse than other coronary stents that may be related to a higher crossing profile, and it must be taken into account when treating severely calcified or tortuous vessels; 2) due to its double-layer design (metallic platform + mesh sleeve fibers), the MGuard stent may compromise side branches, although bifurcation techniques can be performed to recover the compromised side branch; and 3) restenosis rate may be higher than with other commercially available coronary stents.

The MGuard stent can also be used in SVGs when conventional PCI with distal protection devices cannot be performed, as well as to seal coronary perforations that persist despite prolonged balloon inflation. In these cases, however, a high rate of repeated revascularization may be expected. 
The new sirolimus-eluting version of the MGuard stent, which is currently under development, is invented to minimize the restenosis rate of the MGuard Prime stent and will probably be available in late 2015 in Europe.

\section{Disclosure}

The authors report no conflicts of interest in this work.

\section{References}

1. Claussen PA, Abdelnoor M, Kvakkestad KM, Eritsland J, Halvorsen S. Prevalence of risk factors at presentation and early mortality in patients aged 80 years or older with ST-segment elevation myocardial infarction. Vasc Health Risk Manag. 2014;10:683-689.

2. van 't Hof AW, Liem A, Suryapranata H, Hoorntje JC, de Boer MJ, Zijlstra F. Angiographic assessment of myocardial reperfusion in patients treated with primary angioplasty for acute myocardial infarction: myocardial blush grade. Zwolle Myocardial Infarction Study Group. Circulation. 1998;97(23):2302-2306.

3. Niccoli G, Kharbanda RK, Crea F, Banning AP. No-reflow: again prevention is better than treatment. Eur Heart J. 2010;31(20):2449-2455.

4. Stone GW, Webb J, Cox DA, et al. Distal microcirculatory protection during percutaneous coronary intervention in acute ST-segment elevation myocardial infarction: a randomized controlled trial. JAMA. 2005; 293(9):1063-1072.

5. Cohen DJ, Murphy SA, Baim DS, et al. Cost-effectiveness of distal embolic protection for patients undergoing percutaneous intervention of saphenous vein bypass grafts: results from the SAFER trial. J Am Coll Cardiol. 2004;44(9):1801-1808.

6. Fernandez-Rodriguez D, Regueiro A, Brugaletta S, et al. Optimization in stent implantation by manual thrombus aspiration in ST-segmentelevation myocardial infarction: findings from the EXAMINATION trial. Circ Cardiovasc Interv. 2014;7(3):294-300.

7. Jolly SS, Cairns JA, Yusuf S, et al. Randomized trial of primary PCI with or without routine manual thrombectomy. NEngl J Med. 2015;372(15): 1389-1398.

8. Stankovic G, Colombo A, Presbitero P, et al. Randomized evaluation of polytetrafluoroethylene-covered stent in saphenous vein grafts: the Randomized Evaluation of polytetrafluoroethylene COVERed stent in Saphenous vein grafts (RECOVERS) Trial. Circulation. 2003;108(1): $37-42$.

9. Romaguera R, Waksman R. Covered stents for coronary perforations: is there enough evidence? Catheter Cardiovasc Interv. 2011;78(2): $246-253$.

10. Costa JR Jr, Abizaid A, Dudek D, Silber S, Leon MB, Stone GW. Rationale and design of the MGuard for acute ST elevation reperfusion MASTER trial. Catheter Cardiovasc Interv. 2013;82(2):184-190.

11. Dziewierz A, Dudek D. Advantages of MGuard coronary stent system. Minerva Cardioangiol. 2012;60(1):33-40.

12. Stone GW, Abizaid A, Silber S, et al. Prospective, randomized, multicenter evaluation of a polyethylene terephthalate micronet meshcovered stent (MGuard) in ST-segment elevation myocardial infarction: the MASTER trial. J Am Coll Cardiol. Epub 2012 Sep 28.

13. Romaguera R, Gomez-Hospital JA, Sanchez-Elvira G, et al. MGuard mesh-covered stent for treatment of ST-segment elevation myocardial infarction with high thrombus burden despite manual aspiration. J Interv Cardiol. 2013;26(1):1-7.

14. Stone GW. The MASTER II trial. Comparison of the MGuard embolic protection stent with standard stent in acute myocardial infarction. Presented at: The International Conference for Innovations Meeting; December; 2014; Tel Aviv, Israel.

15. Piscione F, Danzi GB, Cassese S, et al. Multicentre experience with MGuard net protective stent in ST-elevation myocardial infarction: safety, feasibility, and impact on myocardial reperfusion. Catheter Cardiovasc Interv. 2010;75(5):715-721.
16. Dudek D, Dziewierz A, Rzeszutko L, et al. Mesh covered stent in STsegment elevation myocardial infarction. EuroIntervention. 2010;6(5): 582-589.

17. Dudek D, Dziewierz A, Kleczynski P, et al. Long-term follow-up of mesh-covered stent implantation in patients with ST-segment elevation myocardial infarction. Kardiol Pol. 2014;72(2):140-145.

18. Fernandez-Cisnal A, Cid-Alvarez B, Alvarez-Alvarez B, et al. Real world comparison of the MGuard Stent versus the bare metal stent for ST elevation myocardial infarction (the REWARD-MI study). Catheter Cardiovasc Interv. 2015;85(1):E1-E9.

19. Amoroso G, Vos NS, Van der Heyden JA, et al. A prospective, postmarket study with the Mguard Prime Embolic Protection Stent in STsegment elevation myocardial infarction: The International MGuard Prime Observational Study (IMOS Prime). Catheter Cardiovasc Interv. Epub 2015 Mar 7.

20. Costa RA, Abizaid A, Lotan C, et al. Impact of thrombus burden on outcomes after standard versus mesh-covered stents in acute myocardial infarction (from the MGuard for acute ST elevation reperfusion trial). Am J Cardiol. 2015;115(2):161-166.

21. Dudek D, Brener SJ, Rakowski T, et al. Efficacy of an embolic protection stent as a function of delay to reperfusion in ST-segment elevation myocardial infarction (from the MASTER Trial). Am J Cardiol. 2014; 114(10):1485-1489.

22. Dudek D, Dziewierz A, Brener SJ, et al. Mesh-covered embolic protection stent implantation in ST-segment-elevation myocardial infarction: final 1-year clinical and angiographic results from the MGUARD for acute ST elevation reperfusion trial. Circ Cardiovasc Interv. 2015;8(2): e001484.

23. Lung HL, Huang LH, Lin HC, Shyur SD. Allergic contact dermatitis to polyethylene terephthalate mesh. J Investig Allergol Clin Immunol. 2009;19(2):161-162.

24. Stone GW, Rogers C, Hermiller J, et al. Randomized comparison of distal protection with a filter-based catheter and a balloon occlusion and aspiration system during percutaneous intervention of diseased saphenous vein aorto-coronary bypass grafts. Circulation. 2003;108(5):548-553.

25. Lee MS, Park SJ, Kandzari DE, et al. Saphenous vein graft intervention. JACC Cardiovasc Interv. 2011;4(8):831-843.

26. Kaluski E, Hauptmann KE, Muller R, Tsai S, Klapholz M, Grube E. Coronary stenting with MGuard: first-in-man trial. J Invasive Cardiol. 2008;20(10):511-515.

27. Grube E, Hauptmann KE, Muller R, Uriel N, Kaluski E. Coronary stenting with MGuard: extended follow-up of first human trial. Cardiovasc Revasc Med. 2011;12(3):138-146.

28. Maia F, Costa JR Jr, Abizaid A, et al. Preliminary results of the INSPIRE trial with the novel MGuard stent system containing a protection net to prevent distal embolization. Catheter Cardiovasc Interv. 2010;76(1):86-92.

29. Romaguera R, Gomez-Hospital JA, Cequier A. Novel use of the Mguard mesh-covered stent to treat coronary arterial perforations. Catheter Cardiovasc Interv. 2012;80(1):75-78.

30. Patil P, Sethi A, Kaul U. Stent thrombosis with an aneurysm 7 years after a drug eluting stent implantation. Indian Heart J. 2014;66(2): 216-219.

31. Danzi GB, Pomidossi GA, Casolo F, Centola M, Ferraresi R, Lotan C. A new device to seal large coronary aneurysms: a case report. $J$ Med Case Rep. 2010;4:238.

32. Schofer J. Evaluation of a PET mesh covered stent in patients with carotid artery disease: results of the first in man CARENET trial. Presented at: the Transcatheter Cardiovascular Therapeutics Conference; September; 2014; Washington, DC, USA.

33. Lindefjeld DS, Guarda E, Méndez M, et al. Microvascular coronary flow comparison in acute myocardial infarction angioplasty treated with a mesh covered stent (MGUARD stent) versus bare metal stent: MICAMI-MGUARD. Cardiovasc Revasc Med. 2013;14(1):4-8. 


\section{Publish your work in this journal}

Vascular Health and Risk Management is an international, peerreviewed journal of therapeutics and risk management, focusing on concise rapid reporting of clinical studies on the processes involved in the maintenance of vascular health; the monitoring, prevention and treatment of vascular disease and its sequelae; and the involvement of metabolic disorders, particularly diabetes. This journal is indexed on PubMed Central and MedLine. The manuscript management system is completely online and includes a very quick and fair peer-review system, which is all easy to use. Visit http://www.dovepress.com/ testimonials.php to read real quotes from published authors.

Submit your manuscript here: http://www.dovepress.com/vascular-health-and-risk-management-journal 